\title{
Effects of calcium electroporation, electrochemotherapy, and irreversible electroporation on quality of life and progression-free survival in patients with pancreatic cancer: IREC clinical study
}

\author{
Julia Rudno-Rudzińska ${ }^{1, A-E}$, Wojciech Kielan²,A,B,F, Maciej Guziński ${ }^{1, B}$, Julita Kulbacka ${ }^{3, A, E, F}$ \\ ${ }^{1} 2^{\text {nd }}$ Department of General and Oncological Surgery, Wroclaw Medical University, Poland \\ 2 Department of Radiology, Wroclaw Medical University, Poland \\ ${ }^{3}$ Department of Molecular and Cellular Biology, Wroclaw Medical University, Poland \\ A - research concept and design; $B$ - collection and/or assembly of data; $C$ - data analysis and interpretation; \\ $D$ - writing the article; $E$ - critical revision of the article; $F$ - final approval of the article
}

\section{Address for correspondence}

Julia Rudno-Rudzińska

E-mail: julia.rudno-rudzinska@umed.wroc.pl

Funding sources

This study was supported by the Medical Research Agency, Poland, Study No. 2020/ABM/01/00098/P/02 (PI: Prof. Wojciech Kielan).

Conflict of interest None declared

Received on July 1,2021

Accepted on July 5, 2021

Published online on July 27, 2021

Cite as

Rudno-Rudzińska J, Kielan W, Guziński M, Kulbacka 」. Effects of calcium electroporation, electrochemotherapy, and irreversible electroporation on quality of life and progression-free survival in patients with pancreatic cancer: IREC clinical study. Adv Clin Exp Med. 2021;30(7):765-770. doi:10.17219/acem/139917

DOI

10.17219/acem/139917

Copyright

Copyright by Author(s)

This is an article distributed under the terms of the

Creative Commons Attribution 3.0 Unported (CC BY 3.0)

(https://creativecommons.org/licenses/by/3.0/)

\begin{abstract}
Background. According to the National Cancer Registry, 3486 people (1744 men and 1742 women) were diagnosed with pancreatic adenocarcinoma in Poland in 2018, resulting in 4908 deaths (2396 men and 2512 women). The only chance of successful treatment is through surgical resection, which is possible in only 20-30\% of patients (stage I, II and some stage III cases).The remaining $70-80 \%$ of patients are those with stage III and IV disease, for whom resection is not possible. Mean survival in these patients is approx. 10.4 months (stage III). In the recent decade, an innovative method called electroporation, which involves destabilization of the cell membrane, has been established. This process can be reversible (RE) or irreversible (IRE), and leads to cell death. The ability to change membrane permeability has led to the development of novel methods involving electrochemotherapy (ECT) and calcium electroporation (CaEP) to treat solid tumors.
\end{abstract}

Objectives. In this study, both ECT and CaEP will be used to treat pancreatic cancer patients with poor prognosis. For each patient, the best "therapeutic moment" for the procedure will be selected based on the therapeutic protocol.

Materials and methods. Patients will receive reversible and irreversible electroporation (control armgroup A), CaEP (active arm-group B), or ECT with intravenous and intratumoral administration of bleomycin (active arm-group C) randomized 1:1:1.

Results. The primary endpoints will be progression-free survival (PFS) and patients' quality of life (QOL) assessed using the EORTC-PAN 26 scale. Secondary endpoints will be patient overall survival (OS), body weight, pain level, and levels of biomarkers such as Ca 19-9.

Conclusions. The Irreversible Electroporation, Electrochemotherapy and Calcium electroporation (IREC) study is necessary to examine the safety and efficiency of irreversible electroporation, electrochemotherapy and calcium electroporation in pancreatic cancer treatment.

Key words: pancreatic cancer, electroporation, electrochemotherapy, calcium electroporation 


\section{Introduction}

Pancreatic cancer is a global problem with increasing incidence, high mortality-to-incidence ratio, lack of screening tools, and few effective forms of treatment. ${ }^{1-3}$ The fiveyear survival rate does not exceed than $7-8 \%$ and most patients are diagnosed at stage III or above, which means the tumor cannot be resected. ${ }^{1}$ As a result, this diagnosis is practically a death sentence for the patient. Contemporary treatment regimens for patients with stage III or IV pancreatic cancer are based on systemic treatment with the FOLFIRINOX regimen or regimens based on nabpaclitaxel and gemcitabine. ${ }^{4-7}$ These regimens have been shown to be more effective than the previous regimens in terms of overall survival (OS) by several weeks, and on this basis they are considered superior and registered for clinical use.

Due to the anatomical location of the pancreas, the use of conventional local ablative therapy has not proven effective due to the thermal effect and the possibility of damaging large vessels and bile ducts. One method that has begun to be used in pancreatic cancer treatment is electroporation. ${ }^{8,9}$ This involves the placement of electrodes and the administration of short electrical pulses (up to $100 \mu \mathrm{s}$ ) with electric field strengths in the range of $1500-3000 \mathrm{~V} / \mathrm{cm}$. The cell membrane is either reversibly rearranged, which potentially increases its permeability to drugs, including chemotherapeutics (reversible electroporation - RE), or cell death occurs due to apoptosis (irreversible electroporation - IRE). ${ }^{10-13}$ This method has started to be used in pancreatic, ${ }^{14}$ liver ${ }^{15}$ and prostate cancer treatments. ${ }^{16}$ The IRE has been shown to be effective in palliative treatment of pancreatic cancer. ${ }^{17}$ Notably, it has proved so effective that it has found its way into the British National Institute for Health and Clinical Excellence (NICE) standards for the treatment of melanoma and head and neck cancers. A protocol for its use, in combination with chemotherapy $(\mathrm{CTH})$, is also described in the European Standard Operating Procedures of Electrochemotherapy (ESCOPE) for the treatment of cutaneous and subcutaneous lesions. ${ }^{18}$

In investigations of electroporation, this method has begun to be combined with chemotherapeutics. The efficacy of this method has been demonstrated both in vitro and in vivo, even reducing drug toxicity to healthy cells. ${ }^{19-25}$ This has led to the emergence of the new field of electrochemotherapy (ECT). The drugs used in ECT include bleomycin and cisplatin, which can be administered intravenously or intratumorally. ${ }^{26}$ It has been calculated that the cytotoxicity of chemotherapeutics to tumor cells increases 700-1000 times when used with IRE, along with a reduction in toxicity to the patient. ${ }^{27-33}$

Calcium ion electroporation (CaEP) is another recent advance in the treatment of solid tumors. Calcium is internalized into tumor cells in excessive amounts due to regulatory mechanisms being disrupted by electroporation, resulting in cell necrosis through an adenosine triphosphate (ATP) deficit, which fails to be replenished. ${ }^{34-40}$ It has also been demonstrated that ECT induces an immune response. ${ }^{32}$ Treatment with CaEP and ECT stimulate the immune system to such an extent that it induces an "abscopal effect", i.e., remission of distant lesions not treated with ECT or IRE. ${ }^{22,27-32}$ Moreover, ECT "breaks" chemo-resistance in solid tumors by using a vascular effect and "trapping" the chemotherapeutic agent in the region of the tumor. ${ }^{25,33}$ This concept has been shown to be efficacious and is used as a standard treatment of skin and subcutaneous cancers. In addition, there is currently a study underway to apply this method to colorectal cancers. In the course of our research, we have applied this method to patients with pancreatic cancer, which was published as the first application of its kind in the world. ${ }^{41}$

Bleomycin was selected as the most active chemotherapeutic agent. Originally, belomycin in ECT was used for treatment of head and neck cancer, melanoma, basal cell carcinoma (BCC) skin lesions, squamous cell carcinoma (SCC), and braest cancer. It has since become the standard of care for skin diseases with bleomycin as the recommended chemotherapeutic agent. ${ }^{42-46}$ Other chemotherapeutic agents that have been tested for efficacy in vitro include daunorubicin, doxorubicin, etoposide, paclitaxel, gemcitabine, 5-fluorouracil, carboplatin, and cisplatin. While daunorubicin, etoposide, and paclitaxel were reported to be ineffective in vitro, cisplatin and bleomycin were found to be the most effective. ${ }^{31,47}$ Bleomycin was also found to be the most effective drug in ECT for pancreatic cancer in vitro while also increasing the immune response of the body. ${ }^{38,48,49}$ Furthermore, in an animal model, ECT with bleomycin was shown to be effective in the treatment of pancreatic cancer. ${ }^{21,50}$ The results of studies involving patients with stage III pancreatic cancer are encouraging; however, the number of procedures performed is small, ${ }^{51}$ and the results are similar to studies on the efficacy of ECT for metastatic liver lesions. ${ }^{42,52,53}$

The ECT and CaEP are evolving therapies for which there are few studies involving only a small number of recruited patients. However, these treatments enable personalized medicine, are feasible for older patients, and offer hope of treatment to patients whose prognosis is already poor upon diagnosis. Since 2018, a project entitled "Electrochemotherapy of solid tumors of the gastrointestinal tract: research on the application of electrochemotherapy in pancreatic cancer with unresectable or oligometastatic lesions" has been conducted at the Wroclaw Medical University, Poland, after receiving approval from the Bioethics Committee (approval No. KB-330/2018).

\section{IREC project assumptions}

The project entitled "Effects of calcium electroporation, electrochemotherapy and irreversible electroporation 
(CaEP, ECT and IRE) on quality of life and progression-free survival in patients with pancreatic cancer" aims to answer questions about the efficacy of electroporation (group A) compared with CaEP (group B) and ECT with bleomycin (group C) in patients with unresectable pancreatic cancer. The safety of these 3 treatments will be compared, as well as their efficacy measured as progression-free survival (PFS) and OS. Another important factor being investigated is patients' quality of life (QOL) after surgery, which will be assessed using the EORTC-PAN 26 scale.

If a patient is qualified for the treatment, their data will be entered into the Case Report Form (CRF) system and the procedures will be performed at the $2^{\text {nd }}$ Department of General Surgery and Surgical Oncology of Wroclaw Medical University or at a partner center possessing the required equipment and levels of staff experience on a referral basis. Data on the treatment and hospital stay will be entered into the system. Data will include information regarding both the procedure and hospital stay. The inclusion and exclusion criteria are listed in Table 1. Patients will receive follow-up at the center where the procedure was performed for consultation and imaging examination at 1 month, 3 months and 6 months after treatment, and then every 6 months thereafter. Examinations are permitted as part of the drug program, as well as measurement of hemoglobin $(\mathrm{Hb})$ [g/dL], Ca 19-9 [U/mL], protein levels $[\mathrm{g} / \mathrm{L}]$, and albumin $[\mathrm{mg} / \mathrm{mL}]$. Patients will be assessed as part of a clinical trial, with examinations ending upon patient death or the end of the project.

In order to jointly implement the planned project, collaboration has been established with Dr. Julie Gehl (Department of Clinical Oncology and Palliative Care, Zealand University Hospital, Roskilde, Denmark), who is a pioneer and specialist in CaEP. Doctor Gehl will contribute human and organizational resources to the project under the conditions specified in the agreement or partnership contract.

\section{Study eligibility and IREC inclusion and exclusion criteria}

The study group (Table 1) includes non-pregnant patients over the age of 18 years with unresectable pancreatic cancer (stage III), which represents the largest population at the time of diagnosis, as well as patients who have undergone resection but have had local recurrence. Histopathological confirmation of the malignancy is required, although intraoperative examination is permissible. A lesion infiltrating the superior mesenteric vein exceeding $180 \%$ of the circumference of the celiac artery, superior mesenteric artery, hepatic artery, or aorta is considered unresectable. Furthermore, patients require computed tomography (CT) scans not more than 30 days prior to joining the study. Patients with cardiac arrhythmias or pacemakers/defibrillators are disqualified due to potential device failure and synchrony of the NanoKnife device with the P wave of the electrocardiogram (ECG). Patients allergic to bleomycin or with pulmonary fibrosis are also excluded due to bleomycin administration in group $\mathrm{C}$.

Patients with stage IV disease will not be eligible for the program due to the previous experience of the center and latent frailty syndrome.

\section{Project implementation}

After providing informed consent to participate in this study, patients will be randomized to 3 groups:

- group A: patients undergoing IRE;

- group B: patients undergoing CaEP. After electroporation, patients will be administered an appropriate dose of intratumoral calcium ions $\left(\mathrm{CaCl}_{2}\right)$ calculated with relation to tumor volume; or

- group C: patients undergoing electrochemotherapy with intravenous and intratumoral administration of bleomycin at a dose of $15,000 \mathrm{IU} / \mathrm{m}^{2}$ as a bolus over $30-60 \mathrm{~s}$, approx. 8-28 min before the electroporation procedure, and simultaneously intratumorally at $1000 \mathrm{IU} / \mathrm{mL}$ at a dose of $1000 \mathrm{UI} / \mathrm{cm}^{2}$ in lesions less than $1 \mathrm{~cm}$ and $250 \mathrm{UI} / \mathrm{cm}^{2}$ in lesions larger than $1 \mathrm{~cm}^{3}$.

All procedures will be performed under general anesthesia and following the administration of muscle relaxants to negate potential muscle spasm when the pulse is administered. A single dose of intravenous antibiotics will also be given perioperatively. The procedure will be carried out using the conventional percutaneous method. Due to complaints of postoperative pain, patients will be administered additional epidural anesthesia during the procedure, which

Table 1. Inclusion and exclusion criteria for the IREC project

\begin{tabular}{|c|c|}
\hline Inclusion criteria & Exclusion criteria \\
\hline $\begin{array}{l}\text { - age over } 18 \text { years } \\
\text { - histopathologically confirmed adenocarcinoma of the pancreas (intraoperative examination possible) } \\
\text { - WHO performance status (ECOG) 0, } 1 \text { or } 2 \\
\text { - written informed consent } \\
\text { - lesion defined as unresectable (infiltration of mesenteric vein or portal vein exceeding } 180 \text { degrees or its } \\
\text { thrombosis, infiltration of the hepatic artery, celiac artery or superior mesenteric artery) on abdominal CT } \\
\text { not older than } 30 \text { days (stage III), or patients after resection with local recurrence of the neoplastic process } \\
\text { - tumor size not larger than } 6 \mathrm{~cm} \text { on a CT scan not older than } 30 \text { days } \\
\text { - patients undergoing chemotherapy or patients with a "de novo" diagnosis of pancreatic cancer }\end{array}$ & $\begin{array}{l}\text { - pregnant women } \\
\text { - patients with pacemakers } \\
\text { - patients with rhythms other than sinus } \\
\text { rhythm on ECG } \\
\text { - patients allergic to bleomycin } \\
\text { - patients with pulmonary fibrosis }\end{array}$ \\
\hline
\end{tabular}

WHO - World Health Organization; ECOG - Eastern Cooperative Oncology Group; CT - computed tomography. 
will be maintained for $72 \mathrm{~h}$. In the case of patients with gastrointestinal obstruction or hyperbilirubinemia, palliative procedures (by-pass) will be performed at the same time.

During the hospital stay, patients will be monitored daily for any deterioration in condition by analyzing morphology and Ca19-9, amylase and lipase levels. Patients will be treated with analgesics, anticoagulants and fluid therapy according to the recognized standards and individual patient's needs. Discharge will take place when their clinical condition stabilizes. Following hospital discharge, all patients will be referred to a clinical oncologist for systemic treatment in accordance with the guidelines.

\section{Patient monitoring}

Patients will be assessed as part of the clinical trial for 12 months. Each follow-up examination will consist of:

- physical examination (body weight, body mass index - BMI);

- health assessment according to World Health Organization (WHO);

- pain assessment according to visual analogue scale (VAS);

- QOL according to European Organization for Research and Treatment of Cancer (EORTC) QLQ-PAN 26 scale;

- Hb level;

- total protein level;

- serum albumin level;

- Ca19-9 marker level.

Imaging examinations utilizing abdominal CT or magnetic resonance imaging (MRI) will be performed at 1 month, 3 months, 6 months, and 1 year following treatment. Patient asessment schedule is presented in Table 2. As the majority of systemically treated patients in the drug programs already have imaging examinations scheduled, these can be used as follow-up examinations. If they are not scheduled, imaging will be prescribed by the examiner.

\section{Analysis of the obtained results and conclusions of the IREC study}

The results obtained in this non-commercial clinical trial will be compared with the results of patients who did not receive electroporation for the treatment of pancreatic cancer. Retrospective analyses will be allowed for comparison, but only for the group treated with the same chemotherapeutic regimens used as part of the current standard of care, namely, FOLFIRINOX, gemcitabine and nab-paclitaxel regimens. The PFS and QOL will be compared. One interesting result will be the OS of both groups. At present, the mean OS for stage III pancreatic cancer patients is $8-10$ months from the time of diagnosis. Treatment with electroporation, CaEP or ECT has been performed in the $2^{\text {nd }}$ Department of General Surgery and Surgical Oncology at Wroclaw Medical University in 15 patients at various stages, achieving a mean survival of 26 months from diagnosis; notably, 7 of these patients are still alive.

Three of the primary outcomes are: 1) assessment of improvements in QOL using the EORTC QOL-PAN26 scale; 2) reduction of pain on the VAS; and 3) increases in body weight and protein and albumin levels as indications of patient well-being. Quality of life will be compared between patients who have undergone ablation and those who have not.

A number of secondary studies will be included as part of this project, including the determination of the Ca19-9 marker for predicting the best "therapeutic moment" and the efficacy of electroporation, CaEP and ECT. Groups A, $\mathrm{B}$ and $\mathrm{C}$ will be compared to each other in terms of safety of use and routes of administration of bleomycin and $\mathrm{Ca}^{2+}$ ions. The comparison will include administration safety and dose. Secondary studies will also include any relevant postoperative complications measured on the ClavienDindo scale and the length of hospitalization.

On the basis of data concerning malignant progression (stage, tumor size and tumor location) and co-morbidities

Table 2. Patient assessment chart for the IREC project

\begin{tabular}{|c|c|c|c|c|}
\hline Parameter & 1 month & 3 months & 6 months & 12 months \\
\hline Clinical examination & $x$ & $x$ & $x$ & $x$ \\
\hline BMI & $x$ & $x$ & $x$ & $x$ \\
\hline Body weight [kg] & $x$ & $x$ & $x$ & $x$ \\
\hline Health according to WHO & $x$ & $x$ & $x$ & $x$ \\
\hline Pain according to VAS & $x$ & $x$ & $x$ & $x$ \\
\hline EORTC QLQ-PAN 26 & $x$ & $x$ & $x$ & $x$ \\
\hline Hb level [mg/dL] & $x$ & $x$ & $x$ & $x$ \\
\hline Protein level [g/dL] & $x$ & $x$ & $x$ & $x$ \\
\hline Albumin level $[\mathrm{g} / \mathrm{dL}]$ & $x$ & $x$ & $x$ & $x$ \\
\hline Ca 19-9 market level [U/mL] & $x$ & $x$ & $x$ & $x$ \\
\hline CT/MRI & $x$ & $x$ & $x$ & $x$ \\
\hline
\end{tabular}

BMI - body mass index; WHO - World Health Organization; VAS - visual analogue scale; CT - computed tomography; MRI - magnetic resonance imaging; EORTC - European Organization for Research and Treatment of Cancer. 
using the Charlson Comorbidity Index, we will select a group of patients from those we believe will benefit most from the treatments. Patients will be individually analyzed in terms of the best "therapeutic moment" which, in the future, may be important in relation to new standards of management and the inclusion of this method as part of the treatment regimen for pancreatic cancer.

The effectiveness of the method will be assessed using abdominal MR and/or abdominal CT. The control methods will be compared and the most effective will be selected. The characteristic changes after IRE and their predictive value for OS and PFS will also be assessed. Overall, this study will evaluate the effectiveness and safety of IRE, CaEP and ECT with bleomycin in the treatment of non-resectable pancreatic cancer. It will also address the question of which of these methods is the safest and most effective, and at which point during treatment a patient should be qualified for this procedure. The IREC project may contribute to the inclusion of a new therapeutic method in the treatment regimen for pancreatic cancer.

\section{ORCID iDs}

Julia Rudno-Rudzińska (1) https://orcid.org/0000-0002-4619-7510 Wojciech Kielan (1) https://orcid.org/0000-0001-6116-6504 Maciej Guziński (D) https://orcid.org/0000-0002-9781-2114 Julita Kulbacka (1) https://orcid.org/0000-0001-8272-5440

\section{References}

1. Siegel RL, Miller KD, Jemal A. Cancer statistics, 2018. CA Cancer JClin 2018;68(1):7-30. doi:10.3322/caac.21442

2. Rahib L, Smith BD, Aizenberg R, Rosenzweig AB, Fleshman JM, Matrisian LM. Projecting cancer incidence and deaths to 2030: The unexpected burden of thyroid, liver, and pancreas cancers in the United States. Cancer Res. 2014;74(11):2913-2921. doi:10.1158/0008-5472. CAN-14-0155

3. Quante AS, Ming C, Rottmann M, et al. Projections of cancer incidence and cancer-related deaths in Germany by 2020 and 2030. Cancer Med. 2016;5(9):2649-2656. doi:10.1002/cam4.767

4. Klaiber U, Leonhardt CS, Strobel O, Tjaden C, Hackert T, Neoptolemos JP. Neoadjuvant and adjuvant chemotherapy in pancreatic cancer. Langenbecks Arch Surg. 2018;403(8):917-932. doi:10.1007/s00423-0181724-8

5. Raufi AG, Manji GA, Chabot JA, Bates SE. Neoadjuvant treatment for pancreatic cancer. Semin Oncol. 2019;46(1):19-27. doi:10.1053/j. seminoncol.2018.12.002

6. Murphy JE, Wo JY, Ryan DP, et al. Total neoadjuvant therapy with FOLFIRINOX in combination with losartan followed by chemoradiotherapy for locally advanced pancreatic cancer: A phase 2 clinical trial. JAMA Oncol. 2019;5(7):1020-1027. doi:10.1001/jamaoncol. 2019.0892

7. Venkatesulu BP, Hsieh CE, Sanders KL, Krishnan S. Recent advances in radiation therapy of pancreatic cancer. F1000Res. 2018;7:F1000 Faculty Rev-1931. doi:10.12688/f1000research.16272.1

8. Daniels C, Rubinsky B. Electrical field and temperature model of nonthermal irreversible electroporation in heterogeneous tissues. J Biomech Eng. 2009;131(7):071006. doi:10.1115/1.3156808

9. Lee EW, Chen C, Prieto VE, Dry SM, Loh CT, Kee ST. Advanced hepatic ablation technique for creating complete cell death: Irreversible electroporation. Radiology. 2010;255(2):426-433. doi:10.1148/radiol.10090337

10. Lee EW, Thai S, Kee ST. Irreversible electroporation: A novel imageguided cancer therapy. Gut Liver. 2010;4(Suppl 1):S99-S104. doi:10. 5009/gnl.2010.4.S1.S99

11. Maor E, Rubinsky B. Endovascular nonthermal irreversible electroporation: A finite element analysis. J Biomech Eng. 2010;132(3):031008. doi:10.1115/1.4001035
12. Thompson CB. Apoptosis in the pathogenesis and treatment of disease. Science. 1995;267(5203):1456-1462. doi:10.1126/science.7878464

13. Susin SA, Lorenzo HK, Zamzami N, et al. Molecular characterization of mitochodrial apoptosis-inducing factor. Nature. 1999;397(6718): 441-446. doi:10.1038/17135

14. Lambert L, Horejs J, Krska Z, et al. Treatment of locally advanced pancreatic cancer by percutaneous and intraoperative irreversible electroporation: General hospital cancer center experience. Neoplasma. 2016;63(2):269-273. doi:10.4149/213_150611N326

15. Thomson KR, Cheung W, Ellis SJ, et al. Investigation of the safety of irreversible electroporation in humans. J Vasc Interv Radiol. 2011; 22(5):611-621. doi:10.1016/j.jvir.2010.12.014

16. Onik G, Mikus P, Rubinsky B. Irreversible electroporation: Implications for prostate ablation. Technol Cancer Res Treat. 2007;6(4):295-300. doi:10.1177/153303460700600405

17. Martin RCG, McFarland K, Ellis S, Velanovich V. Irreversible electroporation in locally advanced pancreatic cancer: Potential improved overall survival. Ann Surg Oncol. 2013;20(3 Suppl):S443-S449. doi:10.1245/ s10434-012-2736-1

18. Campana LG, Edhemovic I, Soden D, et al. Electrochemotherapy: Emerging applications technical advances, new indications, combined approaches, and multi-institutional collaboration. Eur J Surg Oncol. 2019:45(2):92-102. doi:10.1016/j.ejso.2018.11.023

19. Gehl J. Electroporation: Theory and methods, perspectives for drug delivery, gene therapy and research. Acta Physiol Scand. 2003;177(4): 437-447. doi:10.1046/j.1365-201X.2003.01093.x

20. Mir LM, Orlowski S, Belehradek J, Paoletti C. Electrochemotherapy potentiation of antitumour effect of bleomycin by local electric pulses. Eur J Cancer Clin Oncol. 1991;27(1):68-72. doi:10.1016/02775379(91)90064-K

21. Girelli R, Prejanò $S$, Cataldo I, et al. Feasibility and safety of electrochemotherapy (ECT) in the pancreas: A pre-clinical investigation. Radiol Oncol. 2015;49(2):147-154. doi:10.1515/raon-2015-0013

22. Falk H, Lambaa S, Johannesen HH, Wooler G, Venzo A, Gehl J. Electrochemotherapy and calcium electroporation inducing a systemic immune response with local and distant remission of tumors in a patient with malignant melanoma: A case report. Acta Oncol. 2017; 56(8):1126-1131. doi:10.1080/0284186X.2017.1290274

23. Zhang Z, Li W, Procissi D, Tyler P, Omary RA, Larson AC. Rapid dramatic alterations to the tumor microstructure in pancreatic cancer following irreversible electroporation ablation. Nanomedicine. 2014;9(8):1181-1192. doi:10.2217/nnm.13.72

24. Serša G, Jarm T, KotnikT, et al. Vascular disrupting action of electroporation and electrochemotherapy with bleomycin in murine sarcoma. Br J Cancer. 2008;98(2):388-398. doi:10.1038/sj.bjc.6604168

25. Serša G, Beravs K, Čemažar M, Miklavčič D, Demsar F. Contrast enhanced MRI assessment of tumor blood volume after application of electric pulses. Electromagn Biol Med. 1998;17(2):299-306. doi:10.3109/15368379809022574

26. Serša G, Cemazar M, Miklavcic D, Chaplin DJ. Tumor blood flow modifying effect of electrochemotherapy with bleomycin. Anticancer Res. 1999;19(5B):4017-4022. PMID:10628347

27. Postow MA, Callahan MK, Barker CA, et al. Immunologic correlates of the abscopal effect in a patient with melanoma. $N$ Engl $J$ Med. 2012;366(10):925-931. doi:10.1056/NEJMoa1112824

28. Caracò C, Mozzillo N, Marone U, et al. Long-lasting response to electrochemotherapy in melanoma patients with cutaneous metastasis. BMC Cancer. 2013;13:564. doi:10.1186/1471-2407-13-564

29. Kunte $C$, Letulé $V$, Gehl J, et al. Electrochemotherapy in the treatment of metastatic malignant melanoma: A prospective cohort study by InspECT. Br J Dermatol. 2017;176(6):1475-1485. doi:10.1111/ bjd. 15340

30. Mir LM, Orlowski S, Poddevin B, Belehradek JJ. Electrochemotherapy tumor treatment is improved by interleukin-2 stimulation of the host's defenses. Eur Cytokine Netw. 1992;3(3):331-334. PMID:1379837

31. Serša G, Čemažar M, Menart V, Gaberc-Porekar V, Miklavčič D. Anti-tumor effectiveness of electrochemotherapy with bleomycin is increased by TNF-a on SA-1 tumors in mice. Cancer Lett. 1997;116(1):85-92. doi:10.1016/S0304-3835(97)00170-5

32. Gerlini G, Di Gennaro P, Borgognoni L. Enhancing anti-melanoma immunity by electrochemotherapy and in vivo dendritic-cell activation. Oncoimmunology. 2012;1(9):1655-1657. doi:10.4161/onci.21991 
33. Kos B, Voigt $P$, Miklavcic D, Moche M. Careful treatment planning enables safe ablation of liver tumors adjacent to major blood vessels by percutaneous irreversible electroporation (IRE). Radiol Oncol. 2015;49(3):234-241. doi:10.1515/raon-2015-0031

34. Carafoli E, Santella L, Branca D, Brini M. Generation, control, and processing of cellular calcium signals. Crit Rev Biochem Mol Biol. 2001; 36(2):107-260. doi:10.1080/20014091074183

35. Berridge MJ, Bootman MD, Roderick HL. Calcium signaling: Dynamics, homeostasis and remodelling. Nat Rev Mol Cell Biol. 2003;4(7):517529. doi:10.1038/nrm1155

36. Frandsen SK, Gissel H, Hojman P, Tramm T, Eriksen J, Gehl J. Direct therapeutic applications of calcium electroporation to effectively induce tumor necrosis. Cancer Res. 2012;72(6):1336-1341. doi:10.1158/ 0008-5472.CAN-11-3782

37. Frandsen SK, Gehl J. Effect of calcium electroporation in combination with metformin in vivo and correlation between viability and intracellular ATP level after calcium electroporation in vitro. PLoSOne. 2017;12(7):e0181839. doi:10.1371/journal.pone.0181839

38. Hansen EL, Sozer EB, Romeo S, Frandsen SK, Vernier PT, Gehl J. Dosedependent ATP depletion and cancer cell death following calcium electroporation, relative effect of calcium concentration and electric field strength. PLoS One. 2015;10(4):e0122973. doi:10.1371/journal. pone. 0122973

39. Calvet CY, Famin D, André FM, Mir LM. Electrochemotherapy with bleomycin induces hallmarks of immunogenic cell death in murine colon cancer cells. Oncoimmunology. 2014;3:e28131. doi:10.4161/onci.28131

40. Plaschke CC, Gehl J, Johannesen $\mathrm{HH}$, et al. Calcium electroporation for recurrent head and neck cancer: A clinical phase I study. Laryngoscope Investig Otolaryngol. 2019;4(1):49-56. doi:10.1002/lio2.233

41. Rudno-Rudzińska J, Kielan W, Guziński M, Płochocki M, Kulbacka J. The first study of irreversible electroporation with calcium ions and chemotherapy in patients with locally advanced pancreatic adenocarcinoma. App/ Sci. 2020;10(15):5163. doi:10.3390/app10155163

42. Mir LM, Gehl J, Sersa G, et al. Standard operating procedures of the electrochemotherapy: Instructions for the use of bleomycin or cisplatin administered either systemically or locally and electric pulses delivered by the Cliniporator TM by means of invasive or noninvasive electrodes. EJC Suppl. 2006;4(4):14-25. doi:10.1016/j.ejcsup. 2006.08.003
43. Campana LG, Mocellin S, Basso M, et al. Bleomycin-based electrochemotherapy: Clinical outcome from a single institution's experience with 52 patients. Ann Surg Oncol. 2009;16(1):191-199. doi:10.1245/ s10434-008-0204-8

44. Gargiulo M, Papa A, Capasso P, Moio M, Cubicciotti E, Parascandolo S. Electrochemotherapy for non-melanoma head and neck cancers: Clinical outcomes in 25 patients. Ann Surg. 2012;255(6):1158-1164. doi:10.1097/SLA.0b013e31824f68b2

45. National Institute for Health and Care Excellence (NICE). Electrochemotherapy for primary basal cell carcinoma and primary squamous cell carcinoma. https://www.nice.org.uk/guidance/ipg478. Published February 26, 2014.

46. National Institute for Health and Care Excellence (NICE). Electrochemotherapy for metastases in the skin from tumours of non-skin origin and melanoma. https://www.nice.org.uk/guidance/ipg446. Published March 27, 2013.

47. Schmidt G, Juhasz-Böss I, Solomayer EF, Herr D. Electrochemotherapy in breast cancer: A review of references. Geburtshilfe Frauenheilkd. 2014;74(6):557-562. doi:10.1055/s-0034-1368538

48. Miklavčič D, Mali B, Kos B, Heller R, Serša G. Electrochemotherapy: From the drawing board into medical practice. Biomed Eng Online. 2014;13(1):29. doi:10.1186/1475-925X-13-29

49. Bimonte S, Leongito M, Granata V, et al. Electrochemotherapy in pancreatic adenocarcinoma treatment: Pre-clinical and clinical studies. Radiol Oncol. 2016;50(1):14. doi:10.1515/raon-2016-0003

50. Jaroszeski MJ, Illingworth P, Pottinger C, Hyacinthe M, Heller R. Electrically mediated drug delivery for treating subcutaneous and orthotopic pancreatic adenocarcinoma in a hamster model. Anticancer Res. 1999;19(2A):989-994. PMID:10368643

51. Granata V, Fusco R, Piccirillo M, et al. Electrochemotherapy in locally advanced pancreatic cancer: Preliminary results. Int J Surg. 2015;18: 230-236. doi:10.1016/j.ijsu.2015.04.055

52. Tafuto S, von Arx C, De Divitiis C, et al. Electrochemotherapy as a new approach on pancreatic cancer and on liver metastases. Int J Surg. 2015;21(Suppl 1):S78-S82. doi:10.1016/j.ijsu.2015.04.095

53. Edhemovic I, Gadzijev EM, Brecelj E, et al. Electrochemotherapy: A new technological approach in treatment of metastases in the liver. Technol Cancer Res Treat. 2011;10(5):475-485. doi:10.7785/tcrt.2012. 500224 